
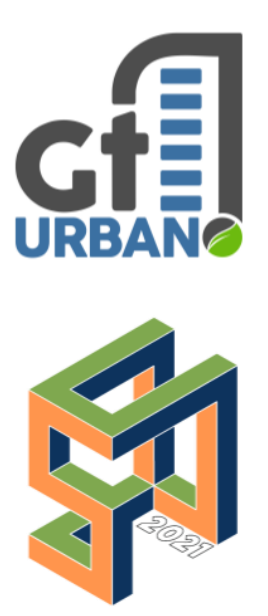

\section{SINGEURB}

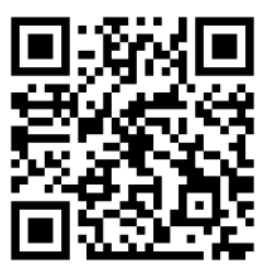

Como citar:

SCHUSSLER, Karina

Rossana Menezes;

MORAES, Odair

Barbosa de;

ZACARIAS;

Assistência técnica

para habitação de

interesse social:

experiências

acadêmicas e

institucionais em

alagoas. In: III

SIMPÓSIO

NACIONAL DE

GESTÃO E

ENGENHARIA

URBANA:

SINGEURB, 2021,

Maceió. Anais...

Porto Alegre: ANTAC,

2021. p. 495-502.

Disponível em:

https://eventos.antac.or

g.br/index.php/singeur

b/issue/view/14

Artigo Compacto

\title{
Assistência técnica para habitação de interesse social: Experiências acadêmicas e institucionais em Alagoas
}

Technical assistance for housing of social interest: Academic and institutional experiences in Alagoas

Karina Rossana Menezes Schüssler, Universidade Estadual de Ciências da

Saúde de Alagoas, karina74rossana@gmail.com

Odair Barbosa de Moraes, Universidade Federal de Alagoas, odair.moraes@gmail.com

Paula Regina Vieira Zacarias, ONU-Habitat, paularvzacarias@gmail.com

\section{RESUMO}

A Assistência Técnica para Habitação de Interesse Social (ATHIS) apresenta-se como uma das estratégias capazes de contribuir para o acesso à moradia adequada, por meio da prestação de serviços técnicos de Arquitetura, Urbanismo e Engenharia voltados para a mitigação do déficit e da inadequação habitacional. O presente artigo analisa duas experiências de ATHIS em Alagoas: (i) atuação do grupo de extensão e pesquisa "Qualidade do Ambiente Construído" da Universidade Federal de Alagoas, em especial as atividades realizadas pelo Fórum MALOCA, em Arapiraca; e (ii) parceria institucional entre o Programa das Nações Unidas para os Assentamentos Humanos (ONU-Habitat), o Governo do Estado de Alagoas e o Conselho de Arquitetura e Urbanismo de Alagoas (CAU/AL) para implementação de melhorias habitacionais nas grotas de Maceió. A sistematização e a análise documental das experiências apresentam as contribuições acadêmicas que qualificam futuros profissionais aptos a atuarem na produção social do habitat alagoano e os avanços e os desafios da articulação institucional para a implementação de ATHIS no estado.

Palavras-chave: Assistência técnica, Habitação de Interesse Social, Produção do habitat.

\begin{abstract}
Technical Assistance for Housing of Social Interest (ATHIS) presents itself as one of the strategies capable of contributing to adequate housing access, through the provision of technical services in Architecture, Urbanism and Engineering turned towards housing deficit and inadequacy mitigation. This article analyzes two experiences of ATHIS in Alagoas: (i) the performance of the "Quality of the Built Environment" an extension and research group from Federal University of Alagoas, in particular, the activities carried out by the MALOCA Forum, in Arapiraca; and (ii) the institutional partnership between the United Nations Program for Human Settlements (UN-Habitat), the Government of the State of Alagoas and the Council of Architecture and Urbanism of Alagoas (CAU/AL) to implement housing
\end{abstract}


improvements in the slum ("grotas") of Maceio. The experiences systematization and documental analysis present the academic contributions that qualify future professionals able to work in the social production of the Alagoas habitat, as well as the advances and challenges of institutional articulation for the implementation of ATHIS in the state.

Keywords: Technical assistance, Housing of Social Interest, Habitat production.

\section{INTRODUÇÃO}

Dados da Fundação João Pinheiro (2021) estimam que o déficit habitacional no Brasil corresponde a 5,8 milhões de moradias, destacando o ônus excessivo com aluguel urbano como o seu principal componente, e mais de 24,8 milhões de residências apresentam algum tipo de inadequação (ausência de espaço de armazenamento, inexistência ou precariedade no banheiro e cobertura e piso inadequados).

Diante deste cenário, a Assistência Técnica para Habitação de Interesse Social (ATHIS), estabelecida pela Lei $n^{\mathrm{o}}$ 11.888/2008, apresenta-se como uma das estratégias capazes de fornecer os serviços técnicos necessários para o acesso à moradia adequada, possibilitando em curto prazo, a adequação habitacional, por meio da melhoria, reforma e/ou ampliação da residência, e em médio e longo prazo, a construção de novas unidades para atendimento das demandas familiares em locais adequados (ONU-Habitat, 2020).

Para exemplificar as possibilidades de implementação de ATHIS como um campo de atuação social para acadêmicos e profissionais da arquitetura, urbanismo e engenharia, o presente artigo sistematiza e analisa experiências recentes de ATHIS em Alagoas desenvolvidas no âmbito do(a):

- Fórum MALOCA realizado pelo grupo de extensão e pesquisa "Qualidade do Ambiente Construído", da Universidade Federal de Alagoas, Campus Arapiraca; e

- Parceria institucional do Programa das Nações Unidas para os Assentamentos Humanos (ONUHabitat), o Governo do Estado de Alagoas, por intermédio da Secretaria de Estado da Infraestrutura (SEINFRA) e o Conselho de Arquitetura e Urbanismo de Alagoas (CAU/AL) para apoio técnico às ações de melhorias habitacionais do Programa "Vida Nova nas Grotas".

Essas experiências apresentam as contribuições acadêmicas com o potencial de qualificar futuros profissionais atuantes na produção social do habitat alagoano e os avanços e desafios da articulação institucional para a implementação de ATHIS no estado.

\section{A MORADIA ADEQUADA E A IMPORTÂNCIA DA ATHIS}

A moradia adequada, reconhecida como parte do direito a um nível de vida adequado pela Declaração Universal de Direitos Humanos de 1948 e o Pacto Internacional de Direitos Econômicos, Sociais e Culturais de 1966, abrange o atendimento às condições de acessibilidade, adequação cultural, disponibilidade de serviços, materiais, instalações e infraestrutura, economicidade, habitabilidade, localização e segurança da posse (ONU-Habitat, 2010).

No Brasil, para garantir a moradia adequada como um direito, existem dois instrumentos: o Estatuto da Cidade, importante marco para política urbana (BRASIL, 2001), e a Lei de ATHIS, fruto da mobilização histórica de diversas entidades de classe profissional que buscavam assegurar a assistência técnica pública e gratuita ao projeto e à construção de Habitação de Interesse Social (HIS) para as famílias de baixa renda (BRASIL, 2008). 
Silvestre e Cardoso (2013) afirmam que o Brasil ganha entre 1 e 1,5 milhão de novas residências por ano, das quais cerca de $70 \%$ são construídas de maneira informal e que cerca de 14 milhões de reformas e ampliações são realizadas anualmente, sendo cerca de 77\% autogeridas. Em 2015, pesquisa realizada pelo Conselho de Arquitetura e Urbanismo do Brasil apontou que menos de 15\% das reformas ou construções utilizavam os serviços de um arquiteto ou engenheiro na obra (CAU/BR, 2015).

Neste sentido, a ATHIS, para além de um direito previsto, representa uma possibilidade de atuação de profissionais das áreas de Arquitetura, Urbanismo e Engenharia na prestação de apoio técnico especializado direcionado à segurança física e estrutural da edificação e às melhores condições de saúde da população.

Diversas iniciativas reforçam a importância da ATHIS como estratégia e campo acadêmico e profissional, a exemplo de: eventos que apresentam atividades de pesquisa, extensão e residência universitária; campanhas e publicações elaboradas por entidades de classe que apontam boas práticas, arranjos institucionais ou modelos de negócios para escritórios populares; e programas de melhorias habitacionais, que servem de exemplos concretos da atuação do poder público em diferentes níveis (CAU/BR, 2021; FNA, 2014; IAB, 2010).

Como contribuição para essas iniciativas, este artigo aborda duas experiências do contexto alagoano, a do Fórum Maloca que se apresenta como o único espaço acadêmico de discussão aberta e periódica sobre HIS em Alagoas, e a parceria do ONU-Habitat, SEINFRA e CAU/AL, como um exemplar de articulação tripartite para garantir o acesso à moradia adequada.

\section{EXPERIÊNCIAS DE ATHIS EM ALAGOAS}

A sistematização das experiências recentes de ATHIS em Alagoas apresenta as contribuições acadêmicas das quatro edições do Fórum MALOCA e os resultados alcançados pela única iniciativa de articulação institucional para a implementação de ATHIS, envolvendo um organismo internacional, o poder público estadual e uma entidade de classe profissional.

\subsection{Experiências acadêmicas}

O Fórum MALOCA consiste em um espaço de discussão sobre HIS, em suas diferentes formas de produção, sendo composto por oficinas, palestras, debates, apresentação de trabalhos, minicursos, exibições audiovisuais e exposições.

Em 2016, com a temática "Perspectivas e conflitos da habitação", a sua primeira edição debateu os problemas habitacionais e as alternativas que poderiam ser utilizadas para reduzir o déficit habitacional (Figura 1). 
Figura 1 - Registro fotográfico do 1o. Fórum Maloca em 2016
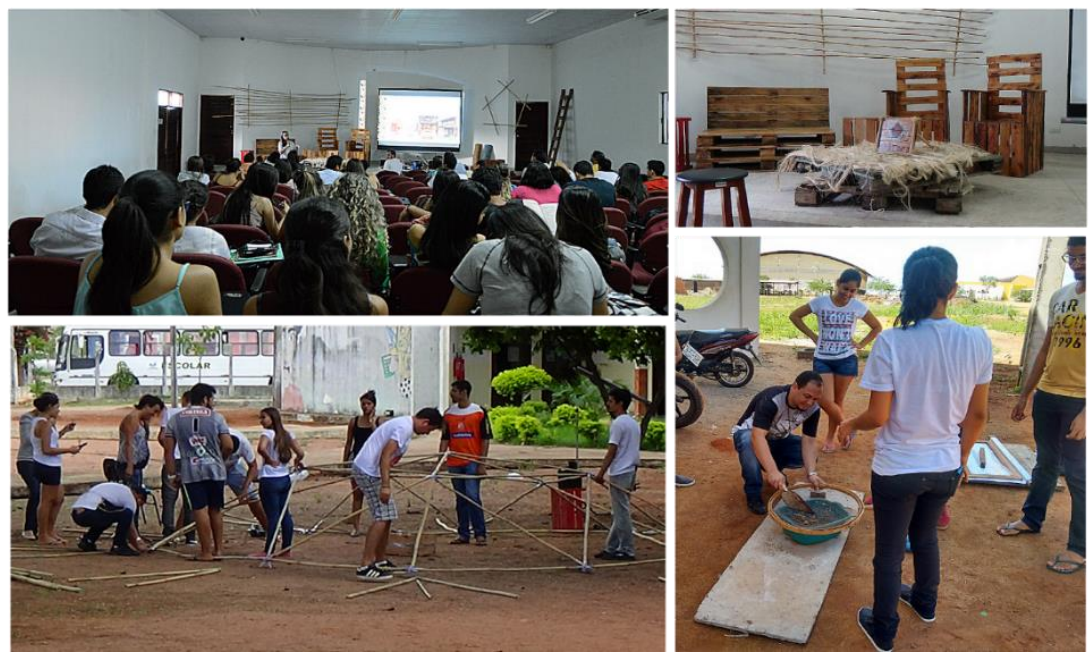

Fonte: Produzidas pelo Autor (2016)

A segunda edição "A produção cotidiana do habitat: da igreja ao cabaré" (2017) apresentou as atividades de extensão realizadas na Igreja de Nossa Senhora do Rosário dos Pretos, em Palmeira dos Índios e de assistência técnica à comunidade do Cabaré Velho, em Arapiraca. Com o tema "O morar entre a legitimidade e a legalidade", em 2018, o Fórum discutiu o cotidiano do morar da população de baixa renda nas cidades brasileiras a partir das transformações sociais e espaciais provocadas pelo Programa Minha Casa, Minha Vida. A última edição "Pelas calçadas sem arquiteto" (2019) contou com o apoio institucional do ONU-Habitat e o apoio técnico da Universidade de Ciências da Saúde do Estado de Alagoas (UNCISAL) e abordou a produção da cidade para além da atuação do Arquiteto e Urbanista, com seus padrões, técnicas e lógicas próprias (Figura 2).

Figura 2 - Registro fotográfico do 4ํㅜ Fórum Maloca em 2019

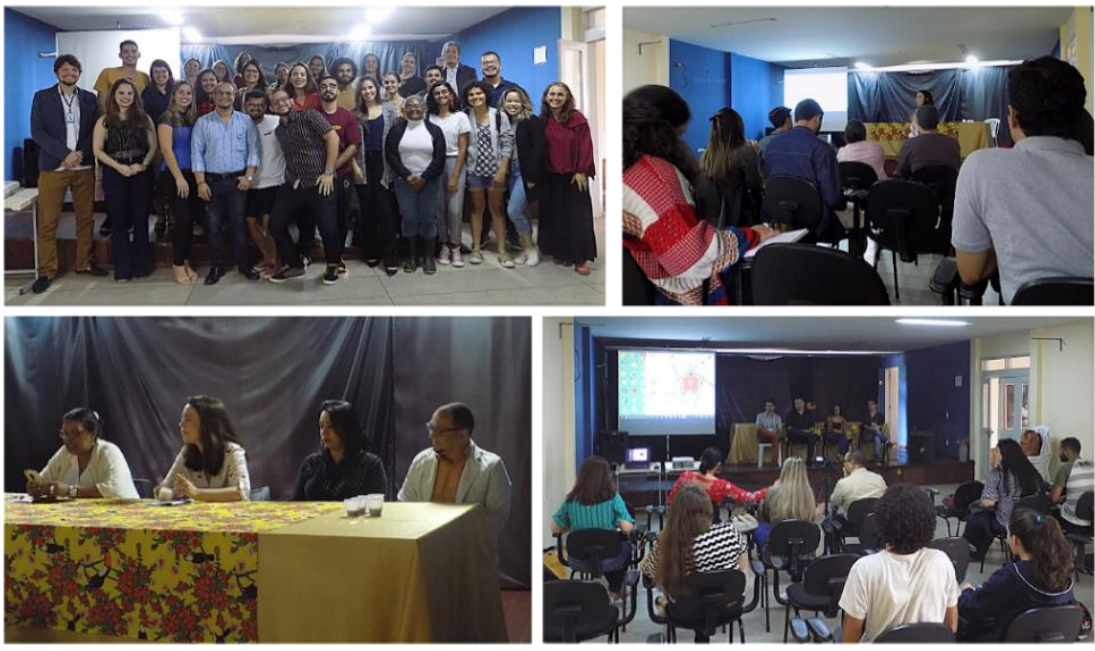

Fonte: Produzidas pelo Autor (2019) 
Dentre os 36 artigos apresentados nas quatro edições, destacam-se cinco que contribuem para a implementação da ATHIS:

- Elaboração do projeto arquitetônico de uma casa modelo sustentável em comparação com o projeto convencional (TEIXEIRA et al, 2016): demonstrou a possibilidade de minimizar os custos da construção de HIS por meio da autoconstrução e da escolha de materiais sustentáveis e recicláveis;

- Relato de experiência sobre assistência técnica prestada à COOPERAGRE de Igaci (CARVALHO et al, 2017): relatou a experiência do MALOCA, enquanto Escritório de Habitação Social, na elaboração de proposta de reforma para a Cooperativa de Crédito Rural do Agreste Alagoano, descrevendo o processo de concepção do projeto e a sua relação com as disciplinas do Curso de Arquitetura e Urbanismo.

- Proposta de Habitação de Interesse Social para a Comunidade Alto da Alegria, Chã Preta-AL (VASCONCELOS NETO; FONSECA, 2019): apresentou projeto de HIS baseado em metodologias de processos participativos;

- Moradia Participativa: A Participação Popular no Processo de Elaboração e Adequação de Projetos Arquitetônicos Habitacionais de Interesse Social (SANTOS; SILVA, 2019): propôs estratégias de planejamento e provisão de HIS a partir de um modelo de gestão denominado Moradia Participativa em dois assentamentos precários de Arapiraca.

- Habitação de Interesse Social no Conjunto Village Campestre II, Maceió-AL (COSTA; FONSECA, 2019): abordou uma metodologia participativa em projetos de HIS para 12 famílias.

A análise destes artigos mostrou um estágio incipiente de produção acadêmica, no entanto, com potencial de desenvolvimento e contribuição na reformulação de disciplinas. Ressalta-se o alinhamento dos trabalhos com princípios chave da ATHIS, pautados em métodos participativos e técnicas alternativas.

\subsection{Experiências institucionais}

Em Maceió, parte dos assentamentos informais da cidade estão localizados nas "grotas" - fundos de vale ambientalmente frágeis e com precárias condições de habitabilidade e infraestrutura. Desde 2016, o Governo de Alagoas executa o Programa "Vida Nova nas Grotas", buscando promover a integração territorial, reduzir as desigualdades socioespaciais e melhorar as condições de vida dos moradores desses assentamentos

As intervenções realizadas pelo Programa já alcançaram 71 grotas com obras de acessibilidade e mobilidade e 11 com ações de melhorias habitacionais, focadas em serviços prioritários (coberta, banheiro, cozinha, fachada, pisos e revestimentos) e complementares (reposições elétricas e hidráulicas).

Para contribuir com essas ações, desde 2018, o ONU-Habitat, a SEINFRA e o CAU/AL atuam em parceria institucional para implementação de ATHIS, tendo realizado 132 projetos de reforma em 8 grotas.

O ONU-Habitat atua com a produção de dados e informações qualificadas sobre as grotas e o compartilhamento de técnicas e metodologias para implantação de projetos de urbanização alinhados aos Objetivos de Desenvolvimento Sustentável (ODS) da Agenda 2030 da ONU e aos princípios da Nova Agenda Urbana (NAU).

A SEINFRA é responsável por executar as ações de melhorias habitacionais com recursos do Fundo Estadual de Combate e Erradicação da Pobreza (FECOEP) e o CAU/AL destina cerca de $6 \%$ do seu 
orçamento para a contratação, por meio de editais de seleção simplificada, de profissionais da Arquitetura e Urbanismo para prestação da assistência técnica (Figura 03) às famílias com renda de até 2 saláriosmínimos.

Figura 3 - Registro fotográfico dos levantamentos técnicos nas grotas de Maceió
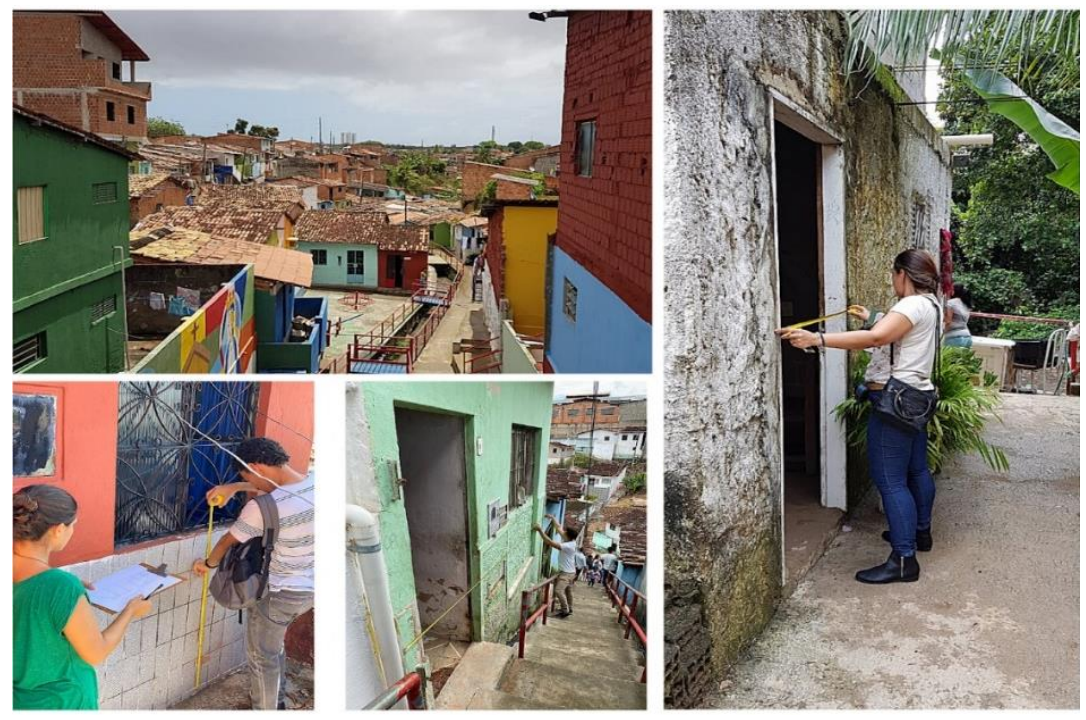

Fonte: Arquivos CAU/AL (2019 e 2020)

\section{CONSIDERAÇÕES FINAIS}

A produção acadêmica do Fórum MALOCA aponta como principais contribuições para a atuação social voltada para a ATHIS: a possibilidade de minimização de custos, por meio da autoconstrução assistida e reutilização de materiais (TEIXEIRA et al, 2016); e o enfoque na cultura de participação e inclusão das comunidades na busca por soluções arquitetônicas adequadas à realidade local (COSTA; FONSECA, 2019).

As lacunas identificadas foram: a ausência de humanização nos projetos existentes, em função do distanciamento entre o apoio técnico e os moradores (VASCONCELOS NETO; FONSECA, 2019); a necessidade de engajamento da população nos processos participativos de elaboração de projetos (SANTOS; SILVA, 2019); e a carência de soluções projetuais integradas com as discussões sobre políticas públicas de ATHIS (SANTOS; SILVA, 2019).

A experiência institucional do ONU-Habitat, SEINFRA e CAU/AL representa os seguintes avanços para Alagoas: a primeira iniciativa estadual de implementação de ATHIS, cumprindo, portanto, as diretrizes da Lei no 11.888/2008 e as recomendações do Plano Estadual de Habitação de Interesse Social, válido até 2020, e do Plano Local de Habitação de Interesse Social de Maceió, finalizado em 2018; a ampliação do número de profissionais qualificados para atuar em assentamentos informais por meio da elaboração de projetos que atendam às necessidades específicas dos moradores; e o aumento da capacidade estatal de execução das melhorias habitacionais (ONU-Habitat, 2020).

Por meio das experiências relatadas, conclui-se que, alguns desafios na ATHIS em Alagoas ainda precisam ser enfrentados: a replicabilidade da articulação, uma vez que envolve a capacidade operacional de instituições com finalidades e portes distintos; a diversificação das fontes de recursos para projetos e obras, 
de modo a aumentar escala e celeridade das ações; e a incorporação de outras entidades e formas de execução das melhorias, abrangendo ONGs, coletivos, escritórios populares, pequenas e médias empresas da construção civil.

As experiências descritas demonstram um espaço de possibilidades para implementação da ATHIS no estado, pois tanto no âmbito acadêmico quanto institucional foram identificadas experiências exitosas, cuja melhor articulação produziria resultados mais eficazes e replicáveis. No entanto, destaca-se o papel e o desempenho crucial do principal agente nessa equação, o poder público, visto que as instituições acadêmicas, profissionais e multilaterais podem contribuir somente na produção social do conhecimento, no assessoramento técnico e no fomento das ações voltadas para garantir o acesso à moradia adequada.

\section{AGRADECIMENTOS}

Os autores agradecem aos graduandos(as) de arquitetura, urbanismo e engenharia que apresentaram suas pesquisas nas quatro edições do Fórum MALOCA, bem como os Arquitetos(as) e Urbanistas atuantes na parceria institucional do ONU-Habitat, SEINFRA e CAU/AL, dado que ambas iniciativas possibilitaram a sistematização e análise das experiências recentes de ATHIS em Alagoas.

\section{REFERÊNCIAS}

BRASIL. Lei no 10.257, de 10 de julho de 2001. Regulamenta os arts. 182 e 183 da Constituição Federal, estabelece diretrizes gerais da política urbana e dá outras providências. Disponível em: <http://www.planalto.gov.br/ccivil_03/leis/leis_2001/110257.htm>. Acesso em: 10 jul. 2021.

BRASIL. Experiências em habitação de interesse social no Brasil. Brasília: Ministério das Cidades, Secretaria Nacional de Habitação, 2007. 219p. Disponível em: <http://www.fna.org.br/wpcontent/uploads/2019/04/FNA-Assist\%C3\%AAncia-T\%C3\%A9cnica-um-direito-de-todos.pdf $>$. Acesso em: 12 jul. 2021.

BRASIL. Lei $\mathrm{n}^{\mathrm{a}}$ 11.888, de 24 de dezembro de 2008. Assegura às famílias de baixa renda assistência técnica pública e gratuita para o projeto e a construção de habitação de interesse social e altera a Lei no 11.124, de 16 de junho de 2005. Disponível em: <http://www.planalto.gov.br/ccivil_03/_ato20072010/2008/lei/111888.htm>. Acesso em: 10 jul. 2021.

CARVALHO, Acácia Bezerra de; SOARES, João Marcos Lira; SANTOS, Larissa Emilly da Silva; LIMA, Lucas Emmanuel; MORAES, Odair Barbosa de. Relato de Experiência Sobre a Assistência Técnica Prestada a COOPERAGRE de Igaci. 2017. In: Site Oficial - Grupo De Extensão e Pesquisa Qualidade do Ambiente Construído - Q-ARA. Disponível em: http://www.q-ara.org/inicio/a-origem. Acesso em 12 jul 2021.

CAU/BR. Campanha Moradia Digna. Conselho de Arquitetura e Urbanismo do Brasil. Brasília: CAU/BR, 2021. Disponível em: <https://caubr.gov.br/moradiadigna/>. Acesso em: 10 jul. 2021.

CAU/BR. Pesquisa Datafolha CAU/BR: O Maior Diagnóstico Sobre Arquitetura e Urbanismo já Feito no Brasil. Conselho de Arquitetura e Urbanismo do Brasil. Brasília: CAU/BR, 2015. Disponível em: $<$ https://www.caubr.gov.br/pesquisa2015/>. Acesso em: 10 jul. 2021. 
COSTA, Jessica Muniz Costa; FONSECA, Débora de Barros Cavalcanti. Habitação de Interesse Social no Conjunto Village Campestre II, Maceió-AL. In: FÓRUM MALOCA, PELAS CALÇADAS SEM ARQUITETO, 4. Anais Fórum Maloca 2019 [...]. Arapiraca: Universidade Federal de Alagoas, 2019. 105 p. E-book (105p.). Tema: Habitação de Interesse Social.

FJP. Déficit Habitacional no Brasil - 2016-2019. Fundação João Pinheiro. Belo Horizonte: FJP, 2021, 169p. Disponível em: <http://novosite.fjp.mg.gov.br/wp-content/uploads/2021/04/21.05_Relatorio-DeficitHabitacional-no-Brasil-2016-2019-v2.0.pdf>. Acesso em : 12 jul. 2021.

FNA. Assistência Técnica e o Direito à Cidade. Federação Nacional dos Arquitetos e Urbanistas, 2014, 97p. Disponível em: <http://www.fna.org.br/wp-content/uploads/2016/06/LivroAT-Internet.pdf>. Acesso em: 12 jul. 2021.

FÓRUM MALOCA, PELAS CALÇADAS SEM ARQUITETO, 4., 2019, Casa da Cultura. Anais Fórum Maloca 2019 [...]. Arapiraca: Universidade Federal de Alagoas, 2019. 105 p. E-book. Tema: Habitação de Interesse Social.

IAB. Manual para a Implantação da Assistência Técnica Pública e Gratuita a Famílias de Baixa Renda para Projeto e Construção de Habitação de Interesse Social. Instituto de Arquitetos do Brasil, 2010, 35p. Disponível em: <https://www.caupr.gov.br/wp-content/uploads/2019/01/manual-para-implantacao-daassistencia-tecnica-publica-e-gratuiata.pdf >. Acesso em: 12 jul. 2021.

ONU-HABITAT. Relatório sobre a implementação de projetos de melhoramento implementados nas grotas. Maceió, 2020. 44p. Disponível em: <https://dados.al.gov.br/catalogo/dataset/grotas-estrategias-urbanas-ehabitacionais>. Acesso em: 12 jul. 2021.

SANTOS, Amanda Cristina; SILVA, Filipe Brian Silva. Moradia Participativa: A Participação Popular No Processo De Elaboração E Adequação De Projetos Arquitetônicos Habitacionais De Interesse Social. In: FÓRUM MALOCA, PELAS CALÇADAS SEM ARQUITETO, 4. Anais Fórum Maloca 2019 [...]. Arapiraca: Universidade Federal de Alagoas, 2019. 105 p. E-book (105p.). Tema: Habitação de Interesse Social.

SILVESTRE, Michelli Garrido; CARDOSO, Luiz Reynaldo de Azevedo. Assistência técnica para melhoria habitacional. Portal Vitruvius, Arquitextos, 157, ano 14, jun. 2013. Disponível em: $<$ https://vitruvius.com.br/revistas/read/arquitextos/14.157/4803>. Acesso em: 12 jul. 2021.

TEIXEIRA, Isabela Vasco; PINA, Arthur Padilha; TORREÃO, Camila Canoa; BORGES, Sávio; FERREIRA, Augusto; ESTEVES, Teresa Alves Abib; CASTRO, Renata Torres S. de. 2016. Elaboração do projeto arquitetônico de uma casa modelo sustentável em comparação com o projeto convencional. In: Site Oficial - Grupo de Extensão e Pesquisa Qualidade do Ambiente Construído - Q-ARA. Disponível em: http://www.q-ara.org/inicio/a-origem. Acesso em 12 jul 2021.

VASCONCELOS NETO, Benedito Soares; FONSECA, Débora de Barros Cavalcanti. Proposta De Habitação De Interesse Social para a Comunidade Alto da Alegria, Chã Preta-AL. In: FÓRUM MALOCA, PELAS CALÇADAS SEM ARQUITETO, 4. Anais Fórum Maloca 2019 [...]. Arapiraca: Universidade Federal de Alagoas, 2019. 105 p. E-book (105p.). Tema: Habitação de Interesse Social. 